\title{
Anticoagulantes nas síndromes coronarianas agudas: uma revisão sistemática
}

\author{
Anticoagulants in acute coronary syndromes: a systematic review
}

Anticoagulantes en síndromes coronarios agudos: una revisión sistemática

Irineu Lopes de Alcântara Júnior ${ }^{1 *}$, Angelo Bruno Pagoto1, Ingrid Loureiro de Queiroz Lima1, Tayrel dos Anjos Silva1', Marcio Jadson Marialva Eliziario¹, Bárbara Aparecida de Souza e Souza ${ }^{1}$, Armando Hiroyuki Mori Júnior ${ }^{1}$, Bárbara Pires Ihara ${ }^{1}$, Irilane de Alcântara Figueira ${ }^{2}$, Ismael Henrique Azevedo de Alcântara².

\section{RESUMO}

Objetivo: Realizar uma revisão sistemática acerca dos anticoagulantes nas síndromes coronarianas agudas (SCA). Métodos: Foi realizada pesquisa em bases eletrônicas em busca de estudos controlados randomizados ou estudos observacionais retrospectivos ou prospectivos que abordassem acerca do uso de anticoagulantes orais em pacientes com síndrome coronariana aguda. A pesquisa incluiu artigos publicados de 2010 a 2019, em inglês ou em português. A seleção ocorreu por dois avaliadores independentes. Resultados: A idade média encontrada foi de mais de 60 anos e a indicação clínica mais comum de anticoagulantes orais foram em pacientes que se submeteram à intervenção coronariana percutânea em decorrência de uma SCA e evoluíram com fibrilação atrial, sendo o mais utilizado a Varfarina. O uso de terapia antitrombótica tripla (anticoagulante + varfarina + clopidogrel) esteve associado a aumento do risco de sangramento com eficácia semelhante quando comparado com uso de aspirina e clopidogrel isolados. Considerações finais: $O$ uso da terapia tripla deve levar em consideração fatores acerca do risco hemorrágico para que seus benefícios superem os riscos.

Palavras-chave: Terapia antitrombótica tripla, Síndrome coronariana aguda, Anticoagulantes orais, Intervenção coronariana percutânea, Fibrilação atrial.

\begin{abstract}
Objective: Propose a systematic review about anticoagulants in acute coronary syndromes (ACS). Methods: Research was conducted on electronic databases to find randomized controlled studies or retrospective or prospective observational studies that addressed the use of oral anticoagulants in patients with acute coronary syndrome. The research included articles published from 2010 to 2019, in English or Portuguese. The selection was carried out by two independent evaluators. Results: According to the methodological proposal, an average age of over 60 years was found. The most common clinical indication for oral anticoagulants was patients who underwent percutaneous coronary intervention as a result of ACS and evolved with atrial fibrillation, the most used being Warfarin. The use of triple antithrombotic therapy (anticoagulant + warfarin + clopidogrel) was associated with an increased risk of bleeding with similar efficacy when compared to the use of aspirin and clopidogrel alone. Final considerations: The use of triple therapy must take into account factors about the hemorrhagic risk so that its benefits outweigh the risks.
\end{abstract}

Keywords: Triple antithrombotic therapy, Acute coronary syndrome, Oral anticoagulants, Percutaneous coronary intervention, Atrial fibrillation.

1 Universidade Federal do Amazonas (UFAM), Manaus - AM. *E-mail: Irineu.alcantara@gmail.com

2 Universidade do Estado do Pará (UEPA), Santarém - PA. 


\section{RESUMEN}

Objetivo: Realizar una revisión sistemática sobre los anticoagulantes en los síndromes coronarios agudos (SCA). Métodos: Se realizó una investigación en bases de datos electrónicas en busca de estudios controlados aleatorizados o estudios observacionales retrospectivos o prospectivos que abordaran el uso de anticoagulantes orales en pacientes con síndrome coronario agudo. La investigación incluyó artículos publicados de 2010 a 2019, en inglés o portugués. La selección fue realizada por dos evaluadores independientes. Resultados: La edad media encontrada fue superior a 60 años y la indicación clínica más frecuente de anticoagulantes orales fue en pacientes sometidos a intervencionismo coronario percutáneo como consecuencia de SCA y evolucionaron con fibrilación auricular, siendo la más utilizada la warfarina. EI uso de triple terapia antitrombótica (anticoagulante + warfarina + clopidogrel) se asoció con un mayor riesgo de hemorragia con una eficacia similar en comparación con el uso de aspirina y clopidogrel solos. Consideraciones finales: El uso de la triple terapia debe tomar en cuenta factores sobre el riesgo hemorrágico para que sus beneficios superen los riesgos.

Palabras clave: Triple terapia antitrombótica, Síndrome coronario agudo, Anticoagulantes orales, Intervención coronaria percutánea, Fibrilación auricular.

\section{INTRODUÇÃO}

As síndromes coronarianas agudas (SCA) são um conjunto de manifestações clínicas da isquemia miocárdica, principal causa de morte mundial e é representada pela angina instável, infarto agudo do miocárdio sem supradesnivelamento de ST (IAMSS-ST), e o infarto agudo do miocárdio com supradesnivelamento de ST (IAMCS-ST), diferenciação baseada na capacidade da isquemia de causar dano ao miocárdio suficiente para liberar marcadores de injúria cardíaca (SILVA FMF, et al., 2015; FONSECA FAH e IZAR MCO, 2016).

O infarto agudo do miocárdio (IAM) é resultante da ruptura de uma placa aterosclerótica, levando a trombose intraluminal e prejudicando o fluxo sanguíneo distal, esse é o mecanismo de ação da terapia antitrombótica que objetiva impedir a extensão do coágulo (SANTOS FG, et al., 2015).

O uso de medicamentos que alteram a adesão e agregação plaquetária para evitar um segundo evento no IAMCS-ST e no IAMSS-ST já está bem definido. Lorga Filho AM, et al. (2013) destacam que o uso do ácido acetilsalicílico (AAS) reduziu a mortalidade por todas as causas de IAM em $23 \%$ e levou à redução do risco relativo de $29 \%$ na incidência de eventos vasculares.

Outro medicamento bastante consolidado no tratamento do IAM é o Clopidogrel, um derivado tienopiridínico inibidor da adenosina difosfato (ADP), e seu uso foi relacionado à redução de $20 \%$ no risco relativo de eventos cardiovasculares. O uso combinado de ácido acetilsalicílico (AAS) e Copidogrel evidenciou redução de $36 \%$ no desfecho combinado de morte. Outros fármacos do grupo dos inibidores de ADP por bloqueio dos receptores P2Y12 são o Prasugrel e o Ticagrelor, onde ambos os fármacos demonstraram serem superiores na redução do desfecho combinado em comparação com o Clopidogrel (LORGA FILHO AM, et al., 2013).

Um dos anticoagulantes mais utilizados é a Varfarina, uma mistura racêmica de dois isómeros (R e $S$ ) na mesma proporção, sendo que o $S$ é cerca de cinco vezes mais potente. O fígado tem papel fundamental na ação dos mecanismos de coagulação, com a produção de alguns fatores e as proteínas anticoagulantes $\mathrm{C} e$ $\mathrm{S}$, além de ser necessária a vitamina $\mathrm{K}$, como fator de oxidação. O mecanismo de ação da Varfarina é justamente o de afetar a produção das proteínas $\mathrm{C}$ e $\mathrm{S}$, que já possuem uma semivida baixa. Além disso, diminuem-se os fatores VII e posteriormente o X e IX, modificando a reação de coagulação no sentido antitrombótico (MARTINS AF, 2015).

A heparina não-fracionada (HNF) é um poliânion de carga elevada e seu mecanismo de ação resulta da sua ligação com um inibidor da serino protease, a antitrombina. Ela causa inibição da trombina e dos fatores 
de coagulação VIla, IX, X e XI. O seu uso resultou em menor mortalidade em 30 dias, evidenciando redução de $6,3 \%$ (GRILLO TA e MIRANDA RC, 2014).

Outro anticoagulante com uso bem estabelecido é a heparina de baixo peso molecular (HBPM), que esteve associada à redução de $17 \%$ no risco relativo para ocorrência de óbito ou infarto não fatal em 30 dias. As HBPM têm baixa atividade antitrombínica, mas mantém a capacidade de inativar o fator Xa. Dentre desse grupo, se encontram ainda o Fondaparinux - inibe diretamente o fator Xa por meio de ligação seletiva à antitrombina - e a Bivalirudina - inibidor do fator lla - que demonstraram redução do risco adicional, mesmo que moderada, mas ainda possuem nível de evidência e classes de recomendação inferiores aos anticoagulantes de uso já estabelecido na prática clínica (LORGA FILHO AM, et al., 2013; CUTLIP D, et al., 2019).

Dentre as indicações de uso de anticoagulação na prática clínica, principalmente em cardiologia, a Diretriz Brasileira de Antiagregantes Plaquetários e Anticoagulantes em Cardiologia destaca o uso no IAMCS-ST, IAMSS-ST, no acidente vascular cerebral, na fibrilação atrial, na valvopatia, no tromboembolismo venoso e perioperatório de cirurgia cardíaca e não cardíaca (LORGA FILHO AM, et al., 2013).

A questão principal a ser discutida neste trabalho é sobre se os benefícios associados ao uso de anticoagulantes, principalmente na SCA, suplantam os riscos inerentes ao seu uso. Esta é uma questão muita debatida nas literaturas internacionais, relacionando o uso de anticoagulantes a um risco aumentado de sangramento, principalmente nos pacientes que fazem uso de dupla antiagregação plaquetária com AAS e algum inibidor da ADP como o Clopidogrel associado a anticogulação oral (PAIKIN JS, et al., 2010).

Este estudo tem como objetivo realizar revisão sistemática acerca dos anticoagulantes nas síndromes coronarianas agudas objetivando comparar a eficácia e o risco de hemorragia da terapia trombótica tripla com anticoagulantes orais mais dupla antiagregação plaquetária com relação aos usuários apenas de dupla antiagregação plaquetária.

\section{MÉTODOS}

Foram selecionados estudos controlados randomizados ou estudos observacionais retrospectivos ou prospectivos acerca do uso de anticoagulantes orais em pacientes com síndrome coronariana aguda.

Foi realizada busca do descritor "antithrombotic therapy", nas bases eletrônicas: Sistema Online de Busca e Análise de Literatura Médica (MEDLINE), a Scientific Electronic Library Online (SciELO), Biblioteca Virtual de Saúde (BVS) e o Google Acadêmico. Para análise dos descritores mais adequados ao objetivo do estudo, optou-se por utilizar os Descritores em Ciências da Saúde (DeCS). O período de publicação dos artigos foi limitado entre 2010 a 2019. A busca abrangeu três etapas: leitura dos títulos, resumos e textos completos, realizadas de modo independente, por dois avaliadores e em caso de divergência uma terceira opinião foi tomada.

Foram incluídos trabalhos publicados no período de janeiro de 2010 a dezembro de 2019, em português ou inglês e que abordavam sobre o uso da anticoagulação nas síndromes coronarianas agudas. Foram excluídos artigos que não permitiam o acesso na íntegra ou que possuíam dados irrelevantes para o objetivo do estudo. Deste modo, 108 artigos foram selecionados para revisão do texto, dos quais 18 atenderam aos critérios de inclusão neste estudo.

A Figura 1 mostra a utilização das etapas da metodologia PRISMA-P na seleção dos artigos para análise. A identificação inclui as etapas 9 e 10 (seleção das fontes de informação e dos critérios de busca). Na identificação foram encontrados 438 artigos, 199 desses eram repetidos e foram mantidos apenas 239. A etapa de rastreio inclui a etapa 11a (retirar os artigos não envolvidos no escopo da pesquisa através de leitura do título e resumo). No rastreio, foram excluídos 131 artigos, restando 108 artigos. A elegibilidade inclui a etapa 11b (aplicação dos critérios de inclusão). Após essa etapa, 76 artigos foram excluídos por terem dados irrelevantes para o objetivo da pesquisa. Após a aplicação dos critérios restaram 18 artigos para serem utilizados na pesquisa (MOHER E, et al., 2015). 
Figura 1 - Fluxograma de seleção dos artigos conforme a metodologia de PRISMA-P.

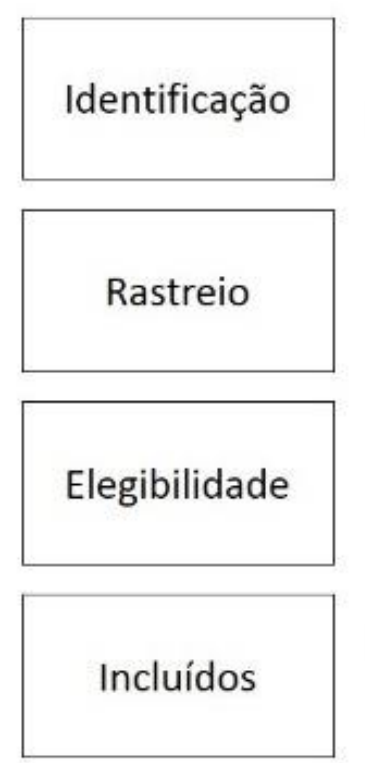

\section{Pesquisa nos bancos de dados 438 artigos encontrados}

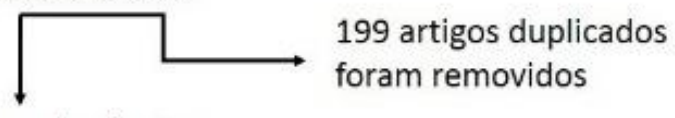

Removidas as duplicatas

239 artigos restantes

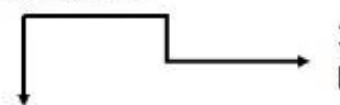

131 artigos excluídos após

Revisão dos títulos e resumos

Aplicar critérios

108 artigos restantes

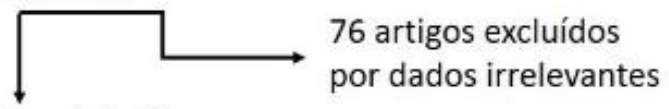

Total de artigos incluídos

18 artigos restantes

Fonte: Alcântara Júnior IL, et al., 2021.

\section{RESULTADOS}

Após a aplicação da metodologia baseada no PRISMA-P, foram selecionados 18 artigos publicados entre 2010 e 2019 para serem discutidos nessa revisão sistemática, relacionado ao uso de anticoagulantes orais em pacientes com SCA que possuem alguma indicação para anticoagulação.

O Quadro 1 está organizado de forma a expor o autor e ano da publicação, o tipo de estudo realizado, o tempo médio de seguimento dos pacientes, a idade média dos participantes da pesquisa, o número de participantes no estudo em questão, qual a principal condição associada a indicação de anticoagulantes orais, o principal anticoagulante utilizado representado no grupo intervenção, quais as terapias de antiagregação plaquetária estão sendo utilizadas nos pacientes que não fazem uso de anticoagulantes orais (ACO) representados no grupo controle e a prevalência de anticoagulação nos pacientes do estudo em questão.

A taxa de trombóticos dos pacientes dos pacientes que fazem uso de ACO em comparação aos pacientes que não fazem uso, sendo que esta pode ser semelhante quando não há diferença estatisticamente significante no desfecho entre os grupos, maior quando ocorrem mais eventos trombóticos no grupo que utiliza ACO e menor quando ocorrem menos eventos trombóticos no grupo que utiliza ACO. Como a ocorrência de eventos trombóticos é o desfecho que se pretende evitar ao utilizar este tipo de tratamento, este é grupo que avalia a eficácia da intervenção utilizada.

A taxa de eventos hemorrágicos comparando o grupo dos pacientes que uso da intervenção com os pacientes que fazem uso do controle, sendo que esta pode ser semelhante quando não há diferença estatisticamente significante no desfecho entre os grupos, maior quando ocorrem mais eventos hemorrágicos associados a intervenção (uso da terapia antitrombótica tripla) que ao grupo controle (uso de dupla antiagregação ou terapia dupla), e menor quando ocorrem menos eventos associados à intervenção.

As duas últimas colunas mostram a taxa hemorragia nos usuários de terapia antitrombótica tripla e a taxa de eventos adversos relacionados à trombose nos usuários de terapia tripla. 
Quadro 1 - Dados resumidos obtidos dos artigos pesquisados.

\begin{tabular}{|c|c|c|c|c|c|c|c|c|c|c|c|c|}
\hline Autor & Tipo & $\begin{array}{c}\text { Seguimento } \\
\text { (meses) }\end{array}$ & $\begin{array}{l}\text { Idade } \\
\text { média } \\
\text { (anos) }\end{array}$ & $\begin{array}{c}\text { Número de } \\
\text { participantes }\end{array}$ & $\begin{array}{l}\text { Condição } \\
\text { clínica }\end{array}$ & Intervenção & Controle & $\begin{array}{l}\text { Prevalência de } \\
\text { anticoagulação }\end{array}$ & $\begin{array}{c}\text { Taxa de } \\
\text { eventos } \\
\text { isquêmicos } \\
\text { da } \\
\text { intervenção } \\
\text { em relação } \\
\text { ao controle }\end{array}$ & $\begin{array}{c}\text { Taxa de } \\
\text { eventos } \\
\text { hemorrágicos } \\
\text { da intervenção } \\
\text { em relação ao } \\
\text { controle }\end{array}$ & $\begin{array}{c}\text { Taxa de } \\
\text { hemorragia } \\
\text { no grupo } \\
\text { intervenção }\end{array}$ & $\begin{array}{c}\text { Taxa de } \\
\text { eventos } \\
\text { adversos } \\
\text { no grupo } \\
\text { intervenção }\end{array}$ \\
\hline $\begin{array}{c}\text { Batra G, et al. } \\
\quad(2018\end{array}$ & Prospectivo & 12 & 74 & 7116 & $\mathrm{FA}$ & $A V K$ + DAA & DAA & $16,2 \%$ & Semelhante & Maior & - & - \\
\hline $\begin{array}{l}\text { Choi HI, et al. } \\
\text { (2012) }\end{array}$ & Prospectivo & - & 68 & 711 & $\mathrm{ICP}+\mathrm{FA}$ & $A V K+D A A$ & DAA & $10,5 \%$ & Semelhante & Maior & - & - \\
\hline $\begin{array}{c}\text { Dabrowska M, } \\
\text { et al. (2013) }\end{array}$ & Prospectivo & 12 & 71 & 104 & $\mathrm{ICP}+\mathrm{FA}$ & $A V K+D A A$ & DAA & $42,0 \%$ & Semelhante & Maior & $38,90 \%$ & $11,10 \%$ \\
\hline $\begin{array}{l}\text { De Vechis R, et } \\
\text { al. (2016) }\end{array}$ & Prospectivo & 12 & 73 & 98 & $\mathrm{ICP}+\mathrm{FA}$ & $A V K+D A A$ & DAA & $49,0 \%$ & Semelhante & Semelhante & $10 \%$ & $27 \%$ \\
\hline $\begin{array}{c}\text { Denas G, et al. } \\
(2012)\end{array}$ & Prospectivo & 6 & 65 & 460 & $\mathrm{ICP}$ & $A V K+D A A$ & DAA & $49,8 \%$ & Semelhante & Maior & $9,80 \%$ & $5,20 \%$ \\
\hline $\begin{array}{l}\text { Enomoto Y, et } \\
\text { al. (2014) }\end{array}$ & Prospectivo & 25 & 70 & 1274 & ICP & $A V K$ + DAA & DAA & $5,8 \%$ & Semelhante & Maior & $12 \%$ & - \\
\hline $\begin{array}{l}\text { Fosbol EL, et } \\
\text { al. (2013) }\end{array}$ & Retrospectivo & 12 & 77 & 1648 & ICP & $A V K+D A A$ & DAA & $27,0 \%$ & Semelhante & Maior & $13,50 \%$ & $20,40 \%$ \\
\hline $\begin{array}{c}\text { Hess CN, et al. } \\
(2015)\end{array}$ & Retrospectivo & 12 & 77 & 4959 & ICP & $A V K+D A A$ & DAA & $27,0 \%$ & Semelhante & Maior & - & - \\
\hline $\begin{array}{c}\text { Jang DO, et al. } \\
(2015)\end{array}$ & Prospectivo & 24 & 69 & 367 & $\mathrm{ICP}+\mathrm{FA}$ & $A V K+D A A$ & DAA & $35,7 \%$ & Semelhante & Maior & $34,30 \%$ & $22,10 \%$ \\
\hline $\begin{array}{c}\text { Koskinas KC, } \\
\text { et al. (2016) }\end{array}$ & Prospectivo & 12 & 73 & 8772 & $\mathrm{ICP}+\mathrm{FA}$ & $A V K+D A A$ & DAA & $43,0 \%$ & Semelhante & Semelhante & - & - \\
\hline $\begin{array}{l}\text { Lamberts M, et } \\
\text { al. (2012) }\end{array}$ & Retrospectivo & 12 & 75 & 11480 & $\mathrm{ICP}$ & $A V K$ + DAA & DAA & $13,0 \%$ & Semelhante & Maior & 1,04 & $1,15 \%$ \\
\hline
\end{tabular}




\begin{tabular}{|c|c|c|c|c|c|c|c|c|c|c|c|c|}
\hline Autor & Tipo & $\begin{array}{c}\text { Seguimento } \\
\text { (meses) }\end{array}$ & $\begin{array}{l}\text { Idade } \\
\text { média } \\
\text { (anos) }\end{array}$ & $\begin{array}{c}\text { Número de } \\
\text { participantes }\end{array}$ & $\begin{array}{l}\text { Condição } \\
\text { clínica }\end{array}$ & Intervenção & Controle & $\begin{array}{l}\text { Prevalência de } \\
\text { anticoagulação }\end{array}$ & $\begin{array}{c}\text { Taxa de } \\
\text { eventos } \\
\text { isquêmicos } \\
\text { da } \\
\text { intervenção } \\
\text { em relação } \\
\text { ao controle }\end{array}$ & $\begin{array}{c}\text { Taxa de } \\
\text { eventos } \\
\text { hemorrágicos } \\
\text { da intervenção } \\
\text { em relação ao } \\
\text { controle }\end{array}$ & $\begin{array}{c}\text { Taxa de } \\
\text { hemorragia } \\
\text { no grupo } \\
\text { intervenção }\end{array}$ & $\begin{array}{c}\text { Taxa de } \\
\text { eventos } \\
\text { adversos } \\
\text { no grupo } \\
\text { intervenção }\end{array}$ \\
\hline $\begin{array}{c}\text { Mennun, et al. } \\
\text { (2015) }\end{array}$ & Retrospectivo & 12 & 73 & 859 & $\mathrm{ICP}+\mathrm{FA}$ & $\mathrm{AVK}+\mathrm{DAA}$ & DAA & $43,0 \%$ & Semelhante & Maior & $11 \%$ & $20 \%$ \\
\hline $\begin{array}{l}\text { Nikolsky E, et } \\
\text { al. (2012) }\end{array}$ & Prospectivo & 12 & 62 & 3320 & ICP & $\mathrm{AVK}+\mathrm{DAA}$ & DAA & $3,8 \%$ & Semelhante & Maior & $17 \%$ & - \\
\hline $\begin{array}{l}\text { Rubboli A, et al. } \\
\qquad(2012)\end{array}$ & Prospectivo & 12 & 73 & 632 & $\mathrm{ICP}+\mathrm{FA}$ & $\mathrm{AVK}+\mathrm{DAA}$ & DAA & $32,0 \%$ & Semelhante & Semelhante & $3 \%$ & $27 \%$ \\
\hline $\begin{array}{l}\text { Saint Etienne } \\
\text { C, et al. (2013) }\end{array}$ & ECR & 12 & - & 573 & ICP & $\mathrm{AVK}+\mathrm{DAA}$ & TD & $100,0 \%$ & Semelhante & Maior & $44 \%$ & $17 \%$ \\
\hline $\begin{array}{l}\text { Sambola A, et } \\
\text { al. (2016) }\end{array}$ & Prospectivo & 12 & 75 & 585 & $\mathrm{ICP}+\mathrm{FA}$ & $\mathrm{AVK}+\mathrm{DAA}$ & DAA & $55,9 \%$ & Menor & Maior & $11 \%$ & $0,60 \%$ \\
\hline $\begin{array}{l}\text { Smith J, et al. } \\
(2012)\end{array}$ & Retrospectivo & 12 & 67 & 2423 & FA & $A V K+D A A$ & DAA & $6,6 \%$ & - & Maior & $10 \%$ & - \\
\hline $\begin{array}{c}\text { Yan Y, et al. } \\
(2017)\end{array}$ & Prospectivo & 12 & 70 & 642 & ICP & $A V K+D A A$ & TD & $100,0 \%$ & Menor & Semelhante & $17,00 \%$ & $5,34 \%$ \\
\hline
\end{tabular}

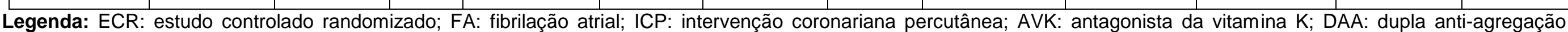
plaquetária com aspirina e clopidogrel; TD: terapia dupla com varfarina e clopidogrel.

Fonte: Alcântara Júnior IL, et al., 2021. 
É possível observar que a maioria $(n=12)$ dos estudos foi conduzido de forma prospectiva, seguido pelos estudos retrospectivos $(n=5)$ e apenas um estudo dentre os encontrados se trata de um estudo controlado randomizado, sendo este o estudo WOEST de Saint Etienne C, et al. (2012).

A maioria dos estudos ( $n=14)$ optou por seguir o desfecho dos pacientes por 12 meses, sendo que o estudo que seguiu por menos tempo o desfecho de seus pacientes foi o estudo de Denas G, et al. (2013), que considerou apenas 6 meses de seguimento em média. $O$ estudo que seguiu por mais tempo seus pacientes foi o estudo de Enomoto Y, et al. (2013), que considerou 25 meses de desfechos clínicos em média.

A idade média dos participantes variou entre 62 anos, no estudo de Nikolsky E, et al., (2012), até 77 anos, nos estudos de Fosbol EL, et al. (2013) e Hess CN, et al. (2015). Com relação ao número de participantes envolvidos em cada pesquisa, este número também foi bastante divergente, sendo de apenas 98 pacientes no estudo de De Verchis R, et al. (2016) até um número de 11480 pacientes (LAMBERTS M, et al., 2012).

A condição clínica que estava associada à indicação de anticoagulantes orais na maioria das pesquisas era a intervenção coronariana percutânea (ICP) nos pacientes com SCA, seguido de fibrilação atrial (FA) nos pacientes com SCA, sendo que em 8 estudos, a ICP e FA estavam presentes nos pacientes. Todos os estudos utilizaram terapia antitrombótica tripla com varfarina, clopidogrel e aspirina. Como grupo controle, foram utilizados aqueles pacientes que não faziam uso de ACO em 16 estudos - sendo usuários apenas da terapia de dupla antiagregação plaquetária com clopidogrel e aspirina -, e os pacientes que faziam uso da terapia antitrombótica dupla em 2 estudos - sendo usuários de varfarina e clopidogrel. Dois estudos compararam o uso da terapia tripla com a terapia dupla (SAINT ETIENNE C, ET AL., 2013; WANG X, ET AL., 2017).

A prevalência da anticoagulação nos pacientes com SCA estudados variou entre $3,8 \%$ no estudo de Nikolsky E, et al. (2012) até 55,9\% no estudo de Sambola A, el al. (2016), levando em consideração apenas os estudos que compararam a terapia tripla com a dupla antiagregação plaquetária. Nos dois estudos que compararam a terapia tripla com a terapia dupla a prevalência de anticoagulação dos pacientes foi $100 \% \mathrm{em}$ razão de todos os pacientes fazerem uso de varfarina, comparando apenas a ausência da aspirina no esquema de tratamento.

Com relação ao desfecho trombótico, a eficácia da terapia tripla em evitar eventos trombóticos foi semelhante à dupla antiagregação plaquetária na maioria dos estudos (14 de 16), sendo que em um dos estudos ela foi considerada menos eficaz e em outro não foi possível encontrar a relação entre a eficácia pela ausência deste dado. Na comparação entre terapia tripla e terapia dupla, a eficácia foi semelhante ou menor da terapia tripla em relação à terapia dupla.

Com relação ao desfecho hemorrágico, a ocorrência de hemorragia foi maior em 13 de 16 estudos que comparavam a terapia tripla com a dupla antiagregação, sendo semelhante no restante dos estudos. $\mathrm{Na}$ comparação entre terapia tripla e terapia dupla, a ausência da varfarina só fez diferença na taxa de hemorragia em um dos dois estudos.

Com relação a taxa de hemorragia e trombose dos usuários de terapia tripla, a obtenção completa desta variável representou uma limitação à análise dos dados, pois os autores não padronizam ou não disponibilizam esta informação, dificultando seu uso correto para comparações. Entretanto, dentre os dados presentes na tabela 1 é possível destacar que a taxa de hemorragia nos pacientes em uso de ACO variou de $3 \%$ até $38,9 \%$ entre os estudos e a taxa de eventos trombóticos variou de $0,6 \%$ a $22,1 \%$ entre os estudos.

A Figura 1 evidencia os sítios de ação de cada fármaco ou classe de fármaco na cascata de coagulação e de adesão/ativação plaquetária. Os vários tipos de anticoagulantes têm eficácia diferente para as variações normais de reações do indivíduo ao medicamento. A cascata de coagulação inicia pela exposição do fator tecidual e termina com a formação de fibrina. Essa cascata pode ser inibida em diferentes pontos por diferentes fármacos, como: antagonistas da vitamina $\mathrm{K}$, epixaban, edoxaban, rivaroxaban, heparina, bivalirudina e dabigatrana. A ação trombótica se inicia na ativação plaquetária, passa pela agregação e termina na formação do trombo, esta fase pode ser inibida pela aspirina, clopidrogrel, prasugrel, ticagrelor, cangrelor e inibidores da GP Ilb/llla (CUTLIP D, et al., 2019). 
Figura 2 - Sítio de ação dos anticoagulantes e antiplaquetários utilizados no tratamento da síndrome coronariana aguda.
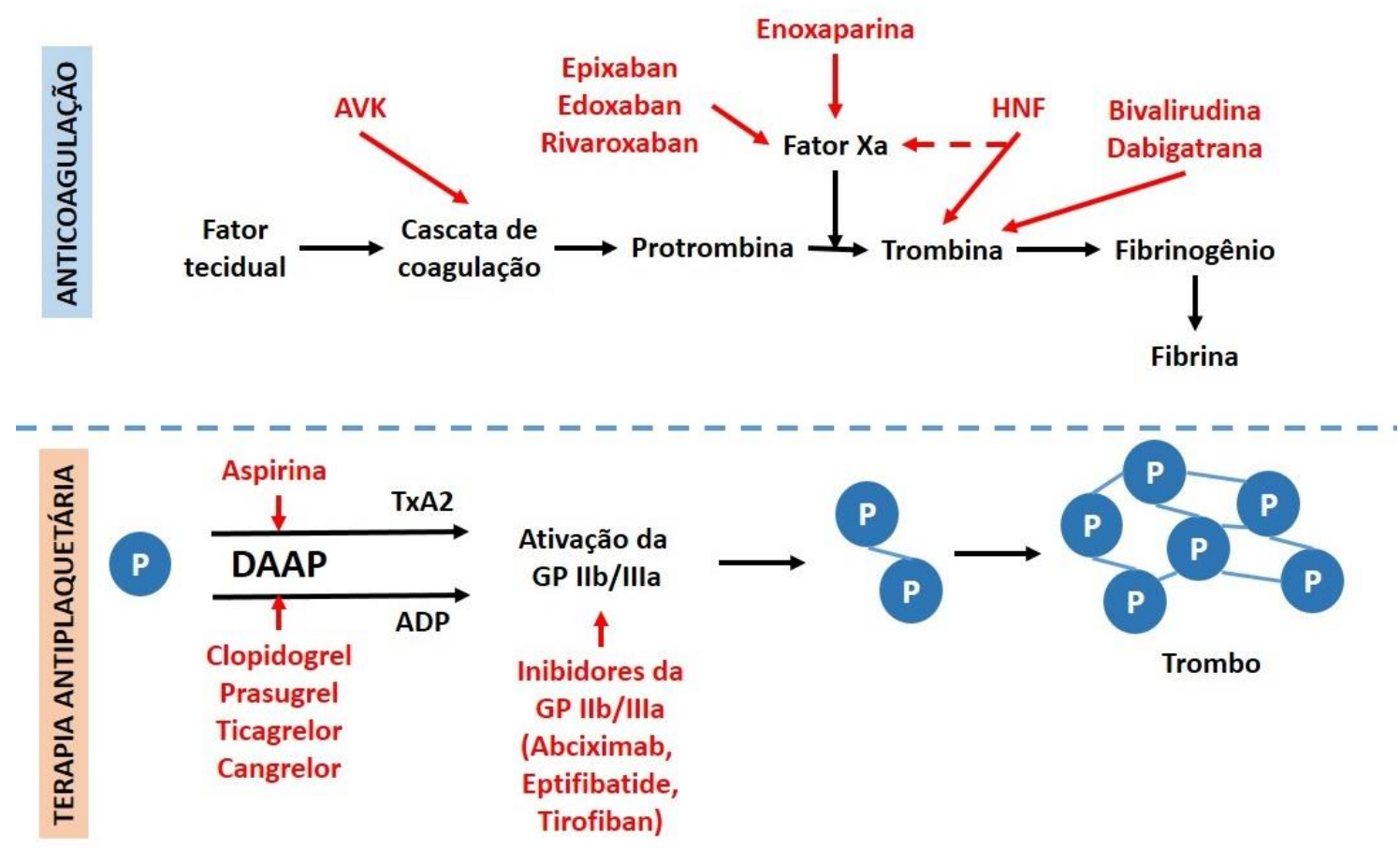

Legenda: AVK: antagonista da vitamina K; HNF: heparina não fracionada; P: plaqueta; DAAP: dupla antiagregação plaquetária; TxA2: tromboxano A2; ADP: adenosina difosfato; GP: glicoproteína. Fonte: Alcântara Júnior IL, et al., 2021. Adaptado de Neumann FJ, et al., 2019.

\section{DISCUSSÃO}

A utilização dos ACO em conjunto com os antiagregantes plaquetários tem vários desafios no que tange a sua utilização. Em todos os estudos a idade foi superior a 60 anos, o que indica que esta é a principal população que utiliza está terapêutica, sendo, portanto, uma população onde o risco de eventos adversos se encontra aumentado devido às comorbidades que geralmente estão presentes. Este fato aponta para a dificuldade que é encontrar recomendações claras acerca do manejo desses pacientes em se tratando de terapia antitrombótica.

A eficácia bem definida dos medicamentos que alteram a adesão e agregação plaquetária tornam o AAS um dos medicamentos mais utilizados para essa finalidade e deve ser levada em consideração durante os estudos. Existem poucas situações clínicas onde o uso do AAS deve ser contraindicado, tal como hipersensibilidade conhecida, úlcera péptica ativa, discrasia sanguínea e hepatopatia grave. Os inibidores da ativação da glicoproteína Ilb/Illa também foram elencados para tratamento do IAMCS-ST, porém com níveis de evidência e classes de recomendação baixa em comparação com outras terapias antiplaquetárias já estabelecidas, o que levanta questões sobre seu uso rotineiro na prática clínica (LORGA FILHO AM, et al., 2013).

Quando se trata da decisão de utilização de um tipo de medicamento ou outro, é importante levar em conta os riscos para os usuários, o que pode incluir tanto o risco hemorrágico quanto o risco trombótico. A partir disso, tem-se que analisar que a idade é um dos fatores de risco para ambas as situações e que, apesar de não ser a mais crucial, pode afetar consideravelmente. No entanto, a idade não deve ser considerada como fator de contraindicação para de ACO ou antiagregante (ZURIMENDI MJA, 2015).

Foi constatado um fato interessante sobre a correlação (IC 95\%, p=0,04) entre a eficiência da terapia de anticoagulação oral e os pacientes com menor funcionalidade, ou seja, maior dependência de cuidados externos. De acordo com Oliveira VP (2017), pacientes com maior dependência física ou verbal tem maior 
índice de eficiência no tratamento, justamente por um fator de auxílio de uma pessoa externa que faz com que as prescrições sejam seguidas à risca, melhorando o quadro do paciente.

Quanto à inserção dos ACO na prática clínica, alguns fatores são levados em consideração durante a elaboração da terapia a ser utilizada. Com o risco elevado de complicações aliada à escassez de dados sobre o desfecho hemorrágico de muitas dessas combinações, o médico acaba optando por prescrever a menor quantidade possível de medicamentos ao elaborar a terapia. Assim como o risco hemorrágico elevado devido o fator idade pode levar o paciente à óbito, o alto custo do tratamento também pode (PEREIRA-DA-SILVA T, et al., 2013).

No cenário intra-hospitalar para pacientes com Síndromes Coronarianas Agudas (SCA), o fator sangramento e hemorrágico são muito preocupantes devido ao possível óbito e morbidade. Por isso, vale ponderar acerca desses pacientes que venham a utilizar ACO ou antiagregantes, visto que com a idade e com a medicação os riscos para os problemas citados aumentam, pode ser mensurado o escore de risco de sangramento, que é um preditivo importante desse âmbito (NICOLAU JC, et al., 2013).

Os estudos evidenciados nos resultados apontaram para uma eficácia semelhante da terapia tripla em relação à dupla antiagregação, este resultado também foi apontado por outros estudos que envolveram o uso de ACO no contexto da SCA associada a FA ou ICP (PAIKIN JS, et al., 2010; ZHU W, et al., 2017). Com relação à maior ocorrência de sangramento, assim como encontrado pelos estudos observacionais pesquisados, outros trabalhos como revisões sistemáticas e meta-análises evidenciaram taxa de sangramento elevado associado a terapia tripla. Este risco foi calculado em até 4 vezes em relação à dupla antiagregação (KERNEIS M, et al., 2018; LAM D, et al., 2018). Estes são resultados importantes, pois evidenciam um risco elevado associado a pouco benefício da terapia tripla em relação à dupla antiagregação.

O trabalho de Paikin J, et al. (2010) encontrou resultados semelhantes com relação a taxa de sangramento da terapia tripla em 1 ano, sendo que esta pode se apresentar em até $12 \%$ dos pacientes deste trabalho em comparação com até 38,9\% encontrado nessa revisão sistemática. Lam D, et al. (2018) também evidenciaram que a terapia dupla em pacientes com FA e que fizeram ICP teve eficácia semelhante à terapia tripla com menos chance de sangramento.

Uma das principais limitações do trabalho foi a ausência de estudos que abordassem a comparação entre os novos anticoagulantes orais (NOACs) e a terapia tripla com varfarina, mostrando a necessidade de mais estudos controlados randomizados nessa temática, haja vista que outros trabalhos já demostram não inferiores dos NOACs em prevenir eventos trombóticos associados a uma menor taxa de sangramento em comparação com a varfarina (PAIKIN J, et al., 2010; LAM D, et al., 2018).

Dentre os NOACs, o Dabigratrana se liga ao sítio ativo da trombina (fator lla) livre e ligada ao coágulo, atua impedindo a atividade pró-coagulante. O uso aumentou o risco de infarto do miocárdio, morte cardíaca ou angina estável em relação ao grupo controle (GRILLO TA, MIRANDA RC, 2014). O Rivaroxabana é um inibidor direto do fator $\mathrm{Xa}$, utilizado pela via oral e é aprovado no Brasil apenas para prevenção de tromboembolismo venoso (TEV) em pacientes adultos submetidos a cirurgia ortopédica de quadril ou joelho. O Apixabana é um potente inibidor oral direto e reversível do fator Xa, é aprovador no Brasil para o mesmo uso do Rivaroxabana e também para prevenção de embolia sistêmica em pacientes com fibrilação atrial (FA) não valvular (GRILLO TA, MIRANDA RC, 2014).

Um dos estudos que compara a eficácia dos novos anticoagulantes orais é o estudo de Lopes RD, et al. (2019), conhecido como AUGUSTUS trial, que compara a taxa de eventos primários e secundários dos pacientes que fizeram uso de apixabana com os pacientes que fizeram uso de antagonista da vitamina $\mathrm{K}$ em paciente com fibrilação atrial que tiveram síndrome coronariana aguda ou foram submetidos a angioplastia. Em relação às complicações hemorrágicas, aos 6 meses, 10,5\% dos pacientes recebendo apixabana tiveram sangramento clínico significante, contra $14,7 \%$ de eventos adversos em pacientes que fizeram uso de varfarina. O que atendeu ao critério de não inferioridade e superioridade.

Com relação a eventos isquêmicos, $6,7 \%$ dos pacientes tiveram morte ou hospitalização por evento isquêmico em relação a $7,1 \%$ de pacientes que fizeram uso de varfarina. Não houve diferença significada 
entre a taxa de eventos isquêmicos comparando os dois tratamentos. Sendo assim, o estudo AUGUSTUS confirma a eficácia e segurança do uso de um NOAC, pois o uso de apixaban foi capaz de causar menos sangramento com os mesmos resultados do uso de varfarina, levando a uma menor taxa de hospitalização por evento secundário (LOPES RD, et al., 2019).

Atualmente, várias pesquisas vêm apontando os NOACs como promissoras opções ao tratamento tradicional. Vários países têm aprovado a sua utilização e, de fato, a comunidade comprova sua grande eficiência, no entanto, como são medicamentos novos, não se sabe quais os efeitos podem causar a longo prazo. Estes medicamentos vêm ganhando destaque nas pesquisas, mas ainda são necessários mais estudos para fortalecer o benefício do uso desses anticoagulantes (FERNANDES ALC, et al., 2015).

\section{CONSIDERAÇÕES FINAIS}

De acordo com a proposta metodológica conclui-se que a idade mais associada ao uso de ACO são pacientes com mais de 60 anos, o ACO mais utilizado na prática clínica é a varfarina. Foi possível estabelecer que o uso da terapia antitrombótica tripla teve eficácia semelhante em reduzir eventos trombóticos que a dupla antiagregação, com maior risco de evento hemorrágico. O estudo foi útil em responder a maioria dos objetivos específicos propostos, com uma limitação moderada em relação a taxa de sangramento e taxa de eventos trombóticos na terapia tripla, devido a heterogeneidade dos artigos encontrados. Foi possível notar outra dificuldade na escassez de estudos controlados randomizados que tratam sobre os NOACs, evidenciando um ponto onde há necessidade de ampliar as pesquisas.

\section{REFERÊNCIAS}

1. ANDRADE PB, BORGES LSR. Antiplaquetários nas síndromes coronarianas agudas. International Journal of Cardiovascular Sciences, 2017; 30(5): 442-451.

2. BATRA G, et al. Antithrombotic therapy after myocardial infarction in patients with atrial fibrillation undergoing percutaneous coronary intervention. European Heart Journal - Cardiovascular Pharmacotherapy, 2018; 4: 36-45.

3. CANNON CP, et al. RE-DUAL PCI Steering Committee and Investigators. Dual antithrombotic therapy with dabigatran after $\mathrm{PCl}$ in atrial fibrillation. N Engl J Med, 2017; 377: 1513-1524.

4. $\mathrm{CHOI} \mathrm{HI}$, et al. Prevalence, Management, and Long-Term (6-Year) Outcomes of Atrial Fibrillation Among Patients Receiving Drug-Eluting Coronary Stents. JACC : Cardiovascular Interventions, 2012; 10(11): 1075-1085.

5. CUTLIP D, et al. Anticoagulant therapy in non-ST elevation acute coronary syndromes. UpToDate. 2019. Disponível em: https://www.uptodate.com/contents/anticoagulant-therapy-in-non-st-elevation-acute-coronary-syndromes. Acesso em: 15 out. 2020.

6. DABROWSKA M, et al. Balancing between bleeding and thromboembolism after percutaneous coronary intervention in patients with atrial fibrillation. Could triple anticoagulant therapy be a solution? Postępy w Kardiologii Interwencyjnej, 2013; 9(3): 234-240.

7. DE VECCHIS R, et al. Clinical Relevance of Anticoagulation and Dual Antiplatelet Therapy to the Outcomes of Patients With Atrial Fibrillation and Recent Percutaneous Coronary Intervention With Stent. J Clin Med Res, 2016; 8(2): 153161

8. DENAS G, et al. Major bleeding in patients undergoing PCl and triple or dual antithrombotic therapy: a parallel-cohort study. J Thromb Thrombolysis, 2013 ; 35: 178-184.

9. ENOMOTO Y, et al. Bleeding risk with triple antithrombotic therapy in patients with atrial fibrillation and drug-eluting stents. Cardiovasc Interv and Ther, 2014, 29(3): 193-199.

10. FERNANDES ALC, et al. Novos anticoagulantes orais (NOACs) na prevenção de acidente vascular encefálico (AVE) e fenômenos tromboembólicos em pacientes com fibrilação atrial. Rev Soc Bras Clin Med, 2015; 13(2): 98-106.

11. FONSECA FAH, IZAR MCO. Fisiopatologia das síndromes coronarianas agudas. Revista da Sociedade Brasileira de Cardiologia do Estado de São Paulo, 2016; 26(2): 74-77.

12. FOSBOL EL, et al. Warfarin use among older atrial fibrillation patients with non-ST-segment elevation myocardial infarction managed with coronary stenting and dual antiplatelet therapy. American Heart Journal, 2013; 166(5): 864870 .

13. GRILLO TA, MIRANDA RC. Os novos anticoagulantes orais na prática clínica. Rev Med Minas Gerais, 2014; 24(Supl 8): 87-95.

14. HESS CN, et al. Use and Outcomes of Triple Therapy Among Older Patients With Acute Myocardial Infarction and Atrial Fibrillation. Journal of the American College of Cardiology, 2015; 66(6) : 616-627.

15. JANG DO, et al. Triple antithrombotic therapy versus dual antiplatelet therapy in patients with atrial fibrillation undergoing drug-eluting stent implantation. Coronary Artery Disease, 2015; 26(5): 372-380. 
16. KERNEIS M, et al. Triple antithrombotic therapy for patients with atrial fibrillation undergoing percutaneous coronary intervention. Progress in Cardiovascular Diseases, 2018; 60(4-5): 524-530.

17. KOSKINAS KC, et al. Duration of Triple Antithrombotic Therapy and Outcomes Among Patients Undergoing Percutaneous Coronary Intervention. JACC : Cardiovascular Intervetions, 2016; 25(9): 1473-1483.

18. LAM D et al. Concomitant Use of Antiplatelets and Anticoagulants in Patients with Coronary Heart Disease and Atrial Fibrillation: What Do Recent Clinical Trials Teach Us? Current Atherosclerosis Report, 2018; 20(1): 1-4.

19. LAMBERTS M, et al. Bleeding After Initiation of Multiple Antithrombotic Drugs, Including Triple Therapy, in Atrial Fibrillation Patients Following Myocardil Infarction and Coronary Intervention A Nationwide Cohort Study. Circulation, 2012; 126(10): 1185-1193.

20. LOPES RD., et al. Antithrombotic Therapy after Acute Coronary Syndrome or PCI in Atrial Fibrillation. New England Journal of Medicine, 2019; 380: 1509-1524.

21. LORGA FILHO AM. Diretrizes Brasileiras de Antiagregantes Plaquetários e Anticoagulantes em Cardiologia. Arquivos Brasileiros de Cardiologia, 2013; 101 (Supl.3): 1-93.

22. MARTINS AF. Novos fármacos anticoagulantes orais alternativos à varfarina. Tese (Doutorado em Ciências Farmacêuticas) - Faculdade de Ciências da Saúde. Universidade Fernando Pessoa, Porto, 2015; 81 p.

23. MENNUN MG. Balancing the Risk of Bleeding and Stroke in Patients With Atrial Fibrillation After Percutaneous Coronary Intervention (from the AVIATOR Registry). The American Journal of Cardiology, 2014; 35: 3155-3179.

24. MOHER D, et al. Preferred reporting items for systematic review and meta-analysis protocols (PRISMA-P) 2015 statement. Systematic Reviews, 2015; 4(1): 1-9.

25. NEUMANN FJ, et al. 2018 ECT/EACTS Guidelines on Myocardial Revascularization. European Heart Journal, 2019; 40(2): 87-165.

26. NICOLAU JC, et al. Diretrizes da Sociedade Brasileira de Cardiologia sobre Angina Instável e Infarto Agudo do Miocárdio sem Supradesnível do Segmento ST (II Edição, 2007) - Atualização 2013/2014. Arq Bras Cardiol, 2014; 102(3): 01-75.

27. NIKOLSKY E, et al. Outcomes of Patients Treated With Triple Antithrombotic Therapy After Primary Percutaneous Coronary Intervention for ST-Elevation Myocardial Infarction (from the Harmonizing Outcomes With Revascularization and Stents in Acute Myocardial Infarction [HORIZONS-AMI] Trial). The American Journal of Cardiology, 2012; 109(6): 831-838.

28. OLIVEIRA VP. Prescrição de anticoagulantes orais e funcionalidade em pacientes idosos portadores de fibrilação atrial em acompanhamento ambulatorial em centro terciário no sul do Brasil (PROJETO AFINA). Dissertação (Mestrado em Cardiologia e Ciências Vasculares) - Faculdade de Medicina. Universidade Federal do Rio Grande do Sul. Porto Alegre, 2017; 57 p.

29. PEREIRA-DA-SILVA T, et al. Impedimentos à Prescrição de Anticoagulação na Fibrilhação Auricular e Atitude Face aos Novos Anticoagulantes Orais. Acta Med Port 2013; 26(2):127-132.

30. PAIKIN JS, et al. Triple Antithrombotic Therapy in Patients With Atrial Fibrillation and Coronary Artery Stents. Circulation, 2010; 121: 2067-2070.

31. RUBBOLI A, et al. Antithrombotic Management and 1-Year Outcome of Patients on Oral Anticoagulation Undergoing Coronary Stent Implantation (from the Registro Regionale Angioplastiche Emilia-Romagna Registry). The American Journal of Cardiology, 2012; 109(10):1411-1417.

32. SAINT ETIENNE C, et al. Antiplatelet therapy strategies after percutaneous coronary intervention in patients needing oral anticoagulation. Future Cardiol, 2013; 9(6) : 759-762.

33. SAMBOLA A, et al. Impact of Triple Therapy in Elderly Patients with Atrial Fibrillation Undergoing Percutaneous Coronary Intervention. PLOS one, 2016; 11(1): e0147245.

34. SANTOS FG, et al. Avaliação da qualidade do atendimento ao paciente com síndrome coronariana aguda no serviço de emergência. Revista Eletrônica de Enfermagem, 2015; 17(4): 1-9.

35. SILVA FMF, et al. Acute management of unstable angina and non-ST segment elevation myocardial infarction. Einstein (São Paulo), 2015; 13(3): 454-461.

36. SMITH J, et al. Triple antithrombotic therapy following an acute coronary syndrome: prevalence, outcomes and prognostic utility of the HAS-BLED score. Eurolntervention, 2012 ; 8: 672-678.

37. WANG $X$, et al. Impact of triple antithrombotic therapy in patients with acute coronary syndrome undergoing percutaneous coronary intervention in real-world practice. Journal of Geriatric Cardiology, 2017; 14: 679-687.

38. YAN $\mathrm{Y}$, et al. Impact of triple antithrombotic therapy in patients with acute coronary syndrome undergoing percutaneous coronary intervention in real-world practice. Journal of Geriatric Cardiology, 2017;14: 679-687.

39. ZHU W, et al. Efficacy and safety of triple versus dual antithrombotic therapy in atrial fibrillation and ischemic heart disease: a systematic review and meta-analysis. Oncotarget, 2017; 14(8): 81154-81166.

40. ZURIMENDI MJA. Uso de anticoagulantes orais em idosos internados em um serviço de medicina. Dissertação (Mestrado em Gerontologia) - Faculdade de Ciências da Saúde. Universidade de Beira Maior. Covilhã, $2015 ; 44$ p. 